\title{
SALUD
}

\section{Prevalencia del hipotiroidismo subclínico en pacientes con síndrome metabólico internados en salas de Clínica Médica del Hospital Regional de Encarnación}

\author{
Marco Antonio Pescador Ruschel ${ }^{1}$, Manuel Esteban Codas ${ }^{2}$
}

\begin{abstract}
Resumen
Introducción: El síndrome metabólico (SM) ha sido relacionado al hipotiroidismo subclínico (HSC) en adultos mayores debido a la fisiopatología de la función tiroidea en el metabolismo de los lípidos y de la glucosa, la presión arterial y la disfunción cardiaca. Varios estudios, entre ellos meta-análisis, concluyeron que el HSC podría estar asociado con un mayor riesgo de SM. La frecuencia de dicha asociación es de 29,32\% en 358 pacientes con síndrome metabólico según un estudio publicado en el Diabetes \& Metabolism Journal en el 2015. Exiguos, pero epidemiológicamente grandes estudios demuestran que el HSC es un problema común, ocurriendo en el 3 al $8 \%$ de la población mundial sin desorden tiroideo conocido y acarrea un riesgo de 2 al 5\% por año de conversión al hipotiroidismo clínico. En el Paraguay, todavía no se han hecho estudios sobre la prevalencia del HSC en el SM.
\end{abstract}

Objetivo: Determinar la prevalencia del HSC en el SM, según edad y sexo, e identificar los componentes metabólicos más frecuentes en estos pacientes.

Material y Método: Se calculó el tamaño de muestra con el programa Epi Info y el método fue transversal y descriptivo. Se alcanzó una muestra de 155 pacientes.

Resultados: Se determinó que hay una prevalencia del 22\% (34) de HSC en pacientes con SM; con una predominancia del 62\% (21) de mujeres y una edad media de 63 años (DE \pm 8 ). La mayoría de los pacientes con HSC (63\%) cumplían con 4 criterios de SM, y la circunferencia abdominal fue el componente más frecuentemente hallado (74\%).

Conclusiones: El estudio concluye que el HSC es altamente prevalente en pacientes con SM. De acuerdo a la epidemiologia local e internacional, los números encontrados en pacientes con SM duplican a los números publicados

\footnotetext{
1. Universidad Nacional de Itapúa. Facultad de Medicina. Departamento de Medicina Interna del Hospital Regional de Encarnación (Residente).

2. Universidad Nacional de Itapúa. Medicina Interna. Facultad de Medicina. Servicio de Clínica Médica del Hospital Regional de Encarnación.

Este trabajo de investigación fue presentado en modalidad póster en la XXVI Jornada de Jóvenes Investigadores en Mendoza, Argentina el 18 de Octubre del 2018, organizado por la Asociación de Universidades Grupo Montevideo.
}

DOI: $10.26885 /$ rcei.foro.2018.142 


\section{Prevalencia del hipotiroidismo subclínico. Pescado \& Codas}

de estudios epidemiológicos grandes en pacientes sin SM. De esta manera se puede determinar que hay una relación evidente entre los componentes metabólicos del SM y el riesgo de un HSC, considerando estos resultados de gran impacto a nivel regional por ser el primer estudio de prevalencia que toca estos temas; sin embargo, se necesita estudios de cohortes con poblaciones más grandes para evaluar la significancia estadística de esta relación. Nuevos estudios se están diseñando actualmente para este efecto a futuro.

Palabras clave: síndrome metabólico, hipotiroidismo subclínico.

\section{Referencias}

Bonora, B. M. \& Fadini, G. P. (2016). Metabolic Syndrome and Related Disorders, 14, 1-3.

Biondi, B. \& Cooper, D. S. (2008). Endocr Rev, 29, 76-1suma31.

Chang, C. H., Yeh, Y. C., Caffrey, J. L., Shih, S. R., Chuang, L. M. \& Tu, Y. K. (2017). Scientific Reports, 7, 6754.

Chang Chia-Hsuin, Yeh Yi-Chun, Caffrey James L, Shih Shyang-Rong, Chuang Lee-Ming, Tu Yu-Kang (2017). Scientific Reports, 7, (7), 6754.

Erdogan, M., Canataroglu, A. \& Ganidagli, S. (2011). J Endocrinol Invest, 34, 48892. doi:10.3275/7202. 